Invited Manuscript, 2014 Pavlovian Research Award Re-revised and resubmitted May 16, 2016

\title{
Higher-order conditioning and the retrosplenial cortex
}

\author{
Travis P. Todd, Roman Huszár, Nicole E. DeAngeli, and David J. Bucci \\ Department of Psychological and Brain Sciences \\ Dartmouth College, Hanover, NH
}

Correspondence:

David J. Bucci, PhD

Dartmouth College

Department of Psychological and Brain Sciences

6207 Moore Hall

Hanover, NH, 03755

david.j.bucci@dartmouth.edu 


\begin{abstract}
The retrosplenial cortex (RSC) is known to contribute to contextual and spatial learning and memory. This is consistent with its well-established connectivity; the RSC is located at the interface of visuo-spatial association areas, and the parahippocampal hippocampal memory system. However, the RSC also contributes to learning and memory for discrete cues. For example, both permanent lesions and temporary inactivation of the RSC have been shown to impair sensory preconditioning, a form of higher-order conditioning. The purpose of the present experiment was to examine the role of the RSC in a closely related higher-order conditioning paradigm: second-order conditioning. Sham and RSC lesioned rats received first-order conditioning in which one visual stimulus (V1) was paired with footshock and one visual stimulus (V2) was not. Following first-order conditioning, one auditory stimulus (A1) was then paired with V1 and a second auditory stimulus (A2) was paired with V2. Although lesions of the RSC impaired the first-order discrimination, they had no impact on the acquisition of secondorder conditioning. Thus, the RSC does not appear necessary for acquisition/expression of second-order fear conditioning. The role of the RSC in higher-order conditioning, as well as a possible dissociation from the hippocampus, is discussed.
\end{abstract}

keywords: fear, second-order, higher-order, retrosplenial, conditioned suppression 


\section{Introduction}

A growing body of research has demonstrated that the retrosplenial cortex (RSC) makes important contributions to spatial and contextual learning and memory (for reviews see Bucci \& Robinson, 2014; Miller, Vedder, Law, \& Smith, 2014; Todd \& Bucci, 2015; Vann, Aggleton, \& Maguire, 2009). For example, the RSC is necessary for acquisition and retrieval of contextual fear conditioning (Corcoran et al., 2011; Keene \& Bucci, 2008a, 2008c; Kwapis et al., 2015), as well as encoding and retrieval of spatial information (Czajkowski et al., 2014). These findings are consistent with the welldocumented visuo-spatial connectivity of the RSC (van Groen \& Wyss, 1990, 1992; 2003), which includes strong reciprocal connections with the visual cortex (e.g., areas 17, 18b) and spatial processing areas such as the anterodorsal thalamic nucleus, postsubiculum, and the entorhinal cortex (van Groen \& Wyss, 1992; Wyss \& van Groen, 1992). Importantly, the RSC also has strong reciprocal connections with regions of the parahippocampal-hippocampal system that are known to be involved in spatial and contextual learning and memory (Sugar, Witter, van Strien, \& Cappaert, 2011; Wyss \& van Groen, 1992).

However, the RSC also contributes to learning and memory for discrete cues in paradigms that are not explicitly spatial or contextual (Gabriel, 1983; Keene \& Bucci, 2008b; Kwapis et al., 2014; 2015; Robinson et al., 2012, 2014). For example, previous experiments from this lab have demonstrated a role for the RSC in sensory preconditioning, a form of higher-order conditioning. In higher-order conditioning procedures, cues gain the ability to elicit conditioned responses (CRs) even though they are never directly paired with reinforcement. For example, in sensory preconditioning 
(e.g., Brogden, 1939), two initially neutral cues (i.e., S2 and S1) are repeatedly presented together (Phase 1) prior to one of the cues (S1) being paired with reinforcement (Phase 2). Subsequently, $\mathrm{S} 2$ elicits a conditioned response (CR) even though it has never been directly paired with the reinforcer (see Figure 1). In sensory preconditioning, responding to S2 indicates that preconditioning established an association between S2 and S1 (e.g., Rescorla \& Cunningham, 1978). Permanent lesions of the RSC, or temporary inactivation of the RSC during Phase 1, prevent sensory preconditioning (Robinson et al., 2012, 2014). These findings indicate that the RSC is necessary for at least some forms of higher-order conditioning.

The purpose of the present experiment was to test the generality of previous studies examining the role of the RSC in higher-order conditioning by extending them in two ways. First, the present experiment examined a different form of higher-order conditioning: second-order conditioning (see Rescorla, 1980). Procedurally, second-order conditioning is nearly identical to sensory preconditioning, with the exception that the order of Phase 1 and Phase 2 are reversed (see Figure 1). In second-order conditioning, S1 is first paired with reinforcement in Phase 1, and S2 is then paired with S1 in Phase 2. As in sensory preconditioning, S2 acquires the ability to elicit a CR even though it was never directly paired with reinforcement. Second, although previous experiments from our laboratory have involved appetitive conditioning paradigms, the current experiment utilized an aversive conditioning paradigm.

The experimental design is depicted in Table 1. Prior to the start of the experiment, rats received either electrolytic or sham lesions of the RSC. Next, all rats received first-order discrimination training with two visual stimuli. One visual stimulus 
(V1+) was paired with footshock, and the second visual stimulus was not (V2-). In the second-order conditioning phase, one auditory cue (A1) was sequentially followed by V1, and a second auditory cue (A2) was followed by V2. Evidence for second-order conditioning would take the form of greater responding to A1 compared to A2. We expected lesions of the RSC to attenuate the acquisition/expression of second-order conditioning for two related reasons. First, the RSC receives input from multiple sensory association areas, making it likely to contribute to learning and memory in situations that involve multiple cues (e.g., Keene \& Bucci, 2008b), such as second-order conditioning. Second, one recent conceptualization of RSC function is that it is specifically involved in conjointly processing and associating sensory stimuli, even in the absence of primary reinforcement (e.g., Bucci \& Robinson, 2014). Thus, both of these related approaches suggest the RSC should contribute to second-order fear conditioning.

\section{Method}

\subsection{Subjects}

The subjects were 16 adult male Long Evans rats, $~ 60$ days old upon arrival, obtained from Harlan Laboratories (Indianapolis, IN). Rats were housed individually and allowed at least 6 days to acclimate to the vivarium with food available ad libitum (Purina standard rat chow; Nestle Purina, St. Louis, MO). Throughout the study, rats were maintained on a 14:10 light-dark cycle and monitored and cared for in compliance with association for Assessment and Accreditation of laboratory Animal Care guidelines and the Dartmouth College Institutional Animal Care and Use Committee. All rats had been previously exposed to three different contexts and had received a total of two $1 \mathrm{~mA}$, 
2-s footshocks in a prior study not reported here. They were naïve to the current configuration of the context (i.e., the combination of visual, tactile, and olfactory cues), lever pressing, and all visual and auditory stimuli used in this experiment.

\subsection{Surgery}

All surgeries took place over a 4-day period that immediately followed the acclimation period. Subjects were anesthetized with isoflurane gas (1.5\% - 3\% in oxygen) and placed in a Kopf stereotaxic apparatus. The skin was retracted and holes were drilled through the skull above each of the intended lesion sites using the rat brain atlas of Paxinos and Watson (2009). Eight rats received bilateral electrolytic lesions (2 mA, $15 \mathrm{~s}$ at each site) of the RSC prior to behavioral training using the stereotaxic coordinates outlined in Table 2. Control rats received sham lesions consisting of a craniotomy and shallow, non-puncturing burr holes to minimize damage to the underlying cortex. Rats were allowed to recover for at least 2 weeks prior to behavioral training. Approximately one month following surgery rats were food restricted to $85 \%$ of their baseline weight.

\subsection{Behavioral apparatus}

The behavioral procedures were carried out in eight identical standard conditioning chambers $(24 \mathrm{~cm} \mathrm{~W} \times 30.5 \mathrm{~cm} \mathrm{~L} \times 29 \mathrm{~cm} \mathrm{H})$. Each chamber (model \# ENV-007, Med Associates, Georgia, VT) was housed in its own sound attenuation chamber (Med Associates, ENV-017M; $66 \mathrm{~cm} \mathrm{~W} \times 56 \mathrm{~cm} \mathrm{H} \times 56 \mathrm{~cm} \mathrm{D)} \mathrm{and} \mathrm{outfitted}$ with an exhaust fan to provide airflow and background noise $(\sim 68 \mathrm{~dB})$. The sidewalls and ceiling were made of clear acrylic plastic and the front and rear walls were made of brushed aluminum. The grid floor was composed of stainless steel rods (5-mm diameter) spaced $1.5 \mathrm{~cm}$ apart (center-to-center). Each chamber was outfitted with a food cup, 
recessed in the center of the front wall. Retractable levers (Med Associates, ENV$112 \mathrm{CM}$ ) were positioned to the left and right of the food cup (the right lever remained retracted throughout the course of the experiment). The chambers were illuminated with a 2.8-W bulb (with a red cover), mounted to the ceiling of the sound attenuating chamber. In addition, there were four panel lights (Med Associates, ENV-221M) in the chamber: one above each lever, one just above the food cup, and one $\sim 16 \mathrm{~cm}$ above the grid floor centered over the food cup. A house-light (Med Associates, ENV-215M) was mounted 24 $\mathrm{cm}$ above the grid floor at the back of the chamber. A speaker (Med Associates, ENV$224 \mathrm{AM}$ ) was located $20 \mathrm{~cm}$ above and to the right of the food cup and was used to present the auditory stimuli.

All visual and auditory stimuli were $30 \mathrm{~s}$ in duration. The center panel light (just above the food cup) and the house light served as the two visual stimuli. During stimulus presentations, the panel light was constantly illuminated, and the house light flashed twice per second. The auditory stimuli were a white noise $(85 \mathrm{~dB})$ and a click $(74 \mathrm{~dB}, 10$ hz) generated by a Med Associates stimulus generator (ANL-926). Footshocks (1 mA, 1 s) were generated by a Med Associates shock generator (ENV-414) for each chamber. The reinforcer was a 45-mg grain-based rodent food pellet (BioServ, Flemington, NJ). The apparatus was controlled by computer equipment located in an adjacent room.

\subsection{Behavioral procedures}

\subsubsection{Baseline lever-press training.}

On the first day of the experiment, each rat was assigned to one chamber and received a single session of magazine training. During this session, food pellets were delivered freely on random-time 60-s (RT 60-s) schedule, resulting in 60 pellets being 
delivered on average. In addition, all lever presses were reinforced with a single pellet. On the following two days, rats received a 1-hour session in which lever responding was reinforced on a random-interval (RI) 15-s schedule. The schedule increased to RI $30 \mathrm{~s}$ for the following two days, and for the next 16 subsequent days the schedule was increased to RI 60 s. During this period some rats with a low rate of responding were maintained on a RI 30 s schedule. However, all rats had at least 5 consecutive days of RI 60 training prior to the pre-exposure phase of the experiment. The RI $60 \mathrm{~s}$ schedule was in place for the remainder of the experiment.

\subsubsection{Pre-exposure.}

All rats then received two days of stimulus pre-exposure. Each stimulus (flashing light $=\mathrm{F}$, steady light $=\mathrm{L}$, noise $=\mathrm{N}$, and click $=\mathrm{C}$ ) was presented twice, for $30 \mathrm{~s}$, over the course of a 1-hr session. The order of stimulus presentation was randomly generated for each day, with the exception that each stimulus had to be presented once in the first 4 trials and once in the last 4 trials. The intertrial interval (ITI) was variable, with an average ITI of 5 minutes $( \pm 25 \%)$.

\subsubsection{First-order conditioning.}

In order to maintain baseline responding during the first-order conditioning phase, all subjects received cycles of 4 days of conditioning followed by 2 days of baseline recovery. During the first 8 sessions ( $~ 1$ hour) of first-order conditioning, all rats received two 30 s presentations of $\mathrm{F}$ and two $30 \mathrm{~s}$ presentations of L. For half of the rats in each group, F was the V1+ and L was the V2-, and for the other rats L was the V1+ and F was the V2-. V1+ was followed by a $1 \mathrm{~mA}, 1 \mathrm{~s}$ footshock, and the V2- was not. The ITI was variable, with a mean of 13.75 minutes $( \pm 25 \%)$. The trial order varied on 
each of the four days. Day 1: V1+, V2-, V1+, V2-, Day 2: V2-, V1+, V2-, V1+, Day 3:

V2-, V1+, V1+, V2-, Day 4: V1+, V2-, V2-, V1+.

At the end of the first 8 sessions of conditioning, there was complete suppression of responding to both cues. Therefore, in order to extinguish responding to the V2-, for the next 16 sessions ( $\sim 1$ hour), each session included $2 \mathrm{~V} 1+$ trials and $6 \mathrm{~V} 2-$ trials. The order of trials was random, with the exception that $1 \mathrm{CS}+$ trial had to occur within the first 4 and the last 4 trials of the session. The ITI was variable, with a mean of 6.8 minutes $( \pm 25 \%)$. There were two sessions of baseline recovery following sessions 12 , 16, and 20. The second-order conditioning phase began the day following session 24 .

\subsubsection{Second-order conditioning.}

There were 4 sessions ( $1 \mathrm{hr}$ ) of second-order conditioning. In all sessions the ITI was variable, with a mean of 6.8 minutes $( \pm 25 \%)$. During the first session, two auditory cues ( $\mathrm{N}$ and $\mathrm{C}$ ) were presented 4 times for $30 \mathrm{~s}$. For half of the rats in each group, $\mathrm{N}$ was immediately followed by the V1+, and C was followed by the V2-, and for the other half

of rats this was reversed. Thus, the identity of the second-order A1 (the auditory stimulus followed by the V1+) and the second-order A2 was fully counterbalanced. No shock was delivered on A1 trials. For the first session, A1 and A2 trials were single alternated, beginning with either A1 or A2 for half of the rats in each group. For sessions 2-4, each session began with a reminder trial of V1 followed by footshock, and V2 followed by no footshock. The order of these reminder trials was randomly generated each day. Following the two reminder trials there were $3 \mathrm{~A} 1$ trials and $3 \mathrm{~A} 2$ trials where the auditory cues were followed by the first-order V1+ and V2-. Trial order was randomly 
generated each session, with the restriction that no more than two A1 or A2 trials could occur consecutively.

\subsubsection{Extinction.}

Following second-order conditioning, there were 8 sessions $(\sim 64 \mathrm{~min})$ of extinction training, where the first-order V1 was repeatedly presented alone. The original V1 was presented 16 times during each session, and no footshocks were delivered.

During extinction, the ITI was variable, with a mean of 3.4 minutes $( \pm 25 \%)$.

\subsection{Behavioral observations and data analysis}

Throughout the experiments, suppression to stimuli was indexed in terms of standard suppression ratios of the form $x /(x+y)$, where $x$ represents the number of lever presses made during the $30 \mathrm{~s}$ stimulus, and $y$ represents the number of lever presses made during the 30 s period just prior to the onset of each conditioned stimulus (pre-CS). Thus, a score of .5 indicates no suppression, whereas a score of 0 indicates complete suppression. Trials were excluded in cases where rats failed to make at least 1 press during the pre-CS period (e.g., Nelson, Sanjuan, Vadillo-Ruiz, Pérez, \& León, 2011). Groups did not differ in the number of trials excluded. Suppression ratios and pre-CS scores were analyzed with analysis of variance (ANOVA) using a rejection region of $p<$ .05 .

\subsection{Lesion verification and analysis}

After the behavioral procedures were completed, rats were deeply anesthetized with an overdose of sodium pentobarbital and transcardially perfused with $0.9 \%$ saline for $2 \mathrm{~min}$, followed by $10 \%$ buffered formalin for $6 \mathrm{~min}$. Coronal brain sections $(60 \mu \mathrm{m})$ were collected using a freezing microtome and were Nissl-stained using thionin. Using a 
compound microscope (Axioskop I, Zeiss, Inc., Thornwood NY), we identified gross tissue damage as necrosis, missing tissue, or marked thinning of the cortex. Outlines of the lesions were drawn onto digital images adapted from Paxinos and Watson (2009) using PowerPoint at 5 levels along the rostro-caudal extent of the RSC (-1.8, -3.0, -4.2, 5.4, and -6.6 mm posterior from bregma). At each level, area measurements were then made with ImageJ, including the total area of the target region and the area of the target region that exhibited gross tissue damage. From these measurements, we report the average percentage of RSC that was damaged. In addition, we report the average percentage of sections across the rostro-caudal plane that exhibited RSC damage ( 20 sections collected for each rat), the average percentage of sections with damage outside the RSC, and the number of rats with damage to regions outside the RSC.

\section{Results}

\subsection{Histology}

Figure 2a shows a photomicrograph of a representative RSC lesion. In Figure 2b, lesion drawings are stacked onto a single atlas image for each of the 5 levels analyzed along the rostro-caudal extent of the RSC. Bilateral RSC damage was observed in all rats, and the average area of RSC damaged on each section analyzed was $63.9 \%($ SEM $=3.8)$. Damage to the RSC was present on all sections collected for each subject, indicating that damage extended throughout the rostro-caudal extent of the RSC. There was minor damage outside the RSC in 7 of 8 rats (e.g., anterior cingulate, visual cortex, motor cortex, cingulum bundle, forceps major corpus callosum). In contrast to RSC damage, the minor damage outside RSC was present on only $28.3 \%(S E M=8.1)$ of sections collected. 
Overall, the extent of RSC damage is entirely consistent with previous studies from our laboratory (e.g., Keene \& Bucci, 2008a, b, c; Robinson et al., 2012).

\subsection{Behavior}

\subsubsection{Baseline.}

Including the magazine training session, there were 21 session of initial lever press training. A 2 (Group) $\times 21$ (Session) ANOVA revealed a main effect of session, $F(20,280)=25.14, p<.01$. Neither the main effect of group nor the group by session interaction were significant, largest $F(20,280)=1.19, p=.25$. During the final session of baseline training, Sham and RSC lesioned rats averaged $13.83(M d n=12.15)$ and 9.90 $(M d n=9.85)$ responses per minute.

\subsubsection{Pre-exposure.}

Unconditioned responding to the 4 stimuli over the two pre-exposure sessions is presented in Figure 3. In general, both Sham and RSC lesioned rats responded similarly. A 2 (Group) $\times 4$ (Stimulus) $\times 2$ (Day) ANOVA revealed a main effect of stimulus, $F(3$, $42)=18.94, p<.01$, and day, $F(1,14)=32.03, p<.01$. There were no other significant main effects or interactions, largest $F(3,42)=2.58, p=.07$.

A parallel analysis examined pre-CS responding, revealing a significant main effect of stimulus, $F(3,42)=5.04, p<.01$, and an interaction between stimulus and group, $F(3,42)=7.53, p<.05$. No other main effects or interactions were significant, largest $F(1,14)=1.86, p=.20$. Simple effect analyses were conducted to identify the source of the interaction between stimulus and group. There were no reliable group differences within any stimulus, largest $F(1,14)=2.49, p=.14$. However, for unknown reasons, Sham lesioned rats responded less in the pre-CS period for the click compared to 
any other stimulus $(p s<.05)$, and more in the pre-CS period for the light compared to the noise $(p<.05)$. For Sham lesioned rats, the mean number of responses made in the preCS period for each stimulus was: 6.91 (Flash, $M d n=7.00$ ), 6.28 (Light, $M d n=6.00$ ), 7.65 (Noise, $M d n=6.75)$, and $5.25($ Click, $M d n=5.00)$. There were no pre-CS differences across stimuli for RSC lesioned rats. For RSC lesioned rats, pre-CS responding for each stimulus was: 5.22 (Flash, $M d n=5.00), 5.72(\mathrm{Light}, M d n=6.13$ ), 5.53 (Noise, $M d n=5.13)$, and $5.21($ Click, $M d n=5.63)$.

\subsubsection{First-order conditioning.}

Suppression to the first-order V1+ and V2- during the first 8 sessions of conditioning is presented in Figure 4. Because pre-CS responding was frequently fully suppressed during these sessions (possibly due to the magnitude of the shock), only the first two trials (one V1+ and one V2-) from each session are presented. Nevertheless, there were still several trials in which pre-CS responding was 0 for rats in both groups, making a repeated measure analysis untenable. We therefore focused our initial analysis on Sessions 1 and 2, in which all rats had a pre-CS score for the first V1+ and V2- trial. A $2($ Group $) \times 2($ Stimulus: V1+ vs. V2-) $\times 2($ Session $)$ ANOVA revealed a main effect of session, $F(1,14)=103.47, p<.01$, and a main effect of group, $F(1,14)=5.35, p<$ .05. In the first two sessions, RSC lesioned rats showed more overall suppression than Sham lesioned rats. No other main effects or interactions were significant, largest $F(1$, $14)=3.46, p=.08$. As shown in Figure 4, suppression was near complete from Session 3 to 8 for both stimuli.

A 2 (Group) $\times 2$ (Stimulus: V1+ vs. V2-) $\times 2($ Session $)$ ANOVA on pre-CS responding did not reveal any significant main effects or interactions, largest $F(1,14)=$ 
$3.72, p=.07$. During the first two sessions, mean responding during the pre-CS period was $6.63(M d n=6.88)$ and $6.00(M d n=5.25)$ for Sham and RSC lesioned rats, respectively.

For Sessions $9-24$, there were $2 \mathrm{~V} 1+$ and $6 \mathrm{~V} 2$ - presentations. These data are presented in Figure 5. It is clear that while both groups remained fully suppressed to V1+, the discrimination between V1+ and V2- was more rapid in the Sham lesioned rats. A 2 $($ Group $) \times 2$ (Stimulus: V1+ vs. V2-) $\times 16($ Session $)$ ANOVA revealed significant main effects of group, stimulus, and session, minimum $F(15,210)=15.39, p<.01$. Additionally, all of the two-way interactions were significant, minimum $F(15,210)=$ $3.88, p<.01$, as well as the three-way interaction between group, stimulus, and session, $F(15,210)=3.84, p<.01$.

To assess the source of the three-way interaction, separate 2 (Group) $\times 16$ (Session) ANOVAs were performed on V1+ and V2-. For V1+, this analysis did not reveal any significant main effects or interactions, largest $F(15,210)=1.21, p=.27$. Thus, there were no differences in responding to V1+ over the course of first-order conditioning. For V2-, this analysis revealed significant main effects of session, group, and an interaction between session and group, minimum $F(15,210)=5.17, p<.01$. As evident from Figure 5, suppression to V2- was slower to extinguish for RSC compared to Sham lesioned rats. For the final session of first-order conditioning, a 2 (Group) $\times 2$ (Stimulus: V1+ vs. V2-) ANOVA revealed a significant main effect of stimulus, $F(1,14)$ $=149.28, p<.01$. No other main effects or interactions were significant, largest $F(1,14)$ $=3.61, p=.08$. Thus, at the end of the first-order conditioning phase, both groups of rats showed more conditioned suppression to V1+ relative to V2-. 
For Sessions $9-24$, pre-CS responding was analyzed with a 2 (Group) $\times 2$ (Stimulus: V1+ vs. V2-) $\times 16$ (Session) ANOVA. This analysis revealed a significant main effect of stimulus, $F(1,14)=11.76, p<.01$, and session, $F(15,210)=9.95, p<.01$. Although the main effect of stimulus was significant, the numerical difference between stimuli was small. Responding in the pre-CS period was $6.20(M d n=5.18)$ and 5.81 $(M d n=4.57)$ for V1+ and V2-, respectively. The session effect likely reflects an increase in pre-CS responding over the course of training. Pre-CS responding averaged 4.44 (Mdn $=4.08)$ and $7.33(M d n=5.96)$, during the $9^{\text {th }}$ and $24^{\text {th }}$ session, respectively. Averaged over sessions 9 to 24, and stimulus V1 and V2, pre-CS responding was $7.04(M d n=6.03)$ and $5.00(M d n=4.09)$ for Sham and RSC lesioned rats, respectively.

During the baseline recovery sessions, Sham and RSC lesioned rats averaged $13.48(M d n=11.78)$ and $9.83(M d n=8.73)$ responses per minute, respectively. The groups did not significantly differ, $F(1,14)=1.91, p=.20$.

\subsubsection{Second-order conditioning.}

The results of the second-order conditioning phase are presented in Figure 6. As the figure suggests, second-order conditioning was observed in both groups. Conditioned suppression to A1 increased over training, whereas conditioned suppression to A2 remained near 0.5 (a ratio of 0.5 indicates no change in lever pressing from the pre-CS to the CS period). A 2 (Group) $\times 2$ (Stimulus: A1+ vs. A2-) $\times 4$ (Session), revealed main effects of stimulus and session, minimum $F(3,42)=10.32, p<.01$. In addition, there were significant two-way interactions between trial and session, and session and group, minimum $F(3,42)=2.86, p<.05$. No other main effects or interactions were significant, largest $F(3,42)=.89, p=.46$. The lack of group effect, or any higher-order interactions 
including both group and stimulus, indicates that second-order conditioning developed similarly for both Sham and RSC lesioned rats. For the first session of second-order conditioning, a 2 (Group) $\times 2$ (Stimulus: A1+vs. A2-) ANOVA did not reveal any significant main effects or interactions, $F(1,14)=.81, p=.38$. For the final session of second-order conditioning, there was a main effect of stimulus $F(1,14)=64.03, p<.01$, indicative of greater suppression to A1 compared to A2. Neither the group effect nor the interaction between group and stimulus were significant, largest $F(1,14)=2.80, p=.12$.

For pre-CS scores during the second-order conditioning phase, a 2 (Group) $\times 2$ (Stimulus: A1 vs. A2) $\times 4$ (Session), did not reveal any significant main effects or interactions, largest $F(1,14)=1.60, p=.23$. Mean responding during the pre-CS period was $7.75(M d n=7.00)$ and $5.62(M d n=4.70)$, for Sham and RSC lesioned rats, respectively.

During the second-order conditioning phase, there were three reminder trials of V1+ and V2-; one each during sessions 2-4. For the three V1+ reminder trials, the average suppression ratio for Sham lesioned rats was .00 $(M d n=.00)$ and for RSC lesioned rats was .02 $(M d n=.00)$. For the three V2- reminder trials, the average suppression ratio for Sham lesioned rats was $.44(M d n=.44)$ and for RSC lesioned rats was .34 $(M d n=.36)$. The groups did not significantly differ on either trial type, largest $F(1,14)=2.33, p=.15$. For $\mathrm{V} 1+$ trials, pre-CS responding averaged $9.16(M d n=9.16)$ and $7.33(M d n=6.00)$ for Sham and RSC lesioned rats, respectively. Likewise, for V2trials, pre-CS responding averaged $7.91(M d n=8.66)$ and $7.03(M d n=5.66)$ for Sham and RSC lesioned rats, respectively. The groups did not differ significantly on either trial type, $F \mathrm{~s}<1$. 
There were also no group differences in responding to V1 on trials where it was paired with A1. For these trials, suppression ratios were calculated using the pre-CS period prior to the onset of A1. The average suppression ratio was $.032(M d n=.00)$ and $.022(M d n=.00)$ for Sham and RSC lesioned rats, respectively, and the groups did not significantly differ, $F<1$. There was, however, more suppression to V2 for RSC lesioned rats than Sham lesioned rats on trials in which V2 was paired with $\mathrm{A} 2, F(1,14)=6.06, p$ $=.03$. The average suppression ratio was $.48(M d n=.46)$ and $.37(M d n=.39)$ for Sham and RSC lesioned rats, respectively. Although suppression was greater to V2 for RSC lesioned rats, this did not produce any differential suppression to A2 (which was reinforced by V2), as noted earlier.

\subsubsection{Extinction.}

Following second-order conditioning, responding to V1 was extinguished. As shown in Figure 7, responding gradually decreased over the course of extinction, and this decrease did not differ between groups. A 2 (Group) $\times 8$ (Session) revealed a main effect of session, $F(7,98)=29.61, p<.01$. Neither the main effect of group, nor the interaction between group and session were significant, largest $F(1,14)=.47, p=.51$. The mean suppression ratio for the final 4 trials of extinction was .46. This did not differ significantly from $0.5, t(15)=1.49, p=.16$, indicating that extinction was complete.

A parallel analysis of pre-CS scores also revealed a main effect of session, $F(7$, $98)=4.45, p<.01$. Again, neither the main effect of group, nor the interaction between group and session were significant, largest $F(7,98)=1.19, p=.31$. Pre-CS responding averaged $6.85(M d n=5.81)$ and $8.31(M d n=6.38)$ for extinction session 1 and 8 , 
respectively. Over the entire extinction phase, pre-CS responding averaged $8.68(M d n=$ 7.44) and $6.40(M d n=4.81)$ for Sham and RSC lesioned rats, respectively.

Finally, we note that all subjects had received two footshocks prior to the start of the current experiment. However, given the fact that all subjects then went on to receive an additional 48 footshocks during first-order conditioning, it is unlikely the initial two significantly influenced the present results. Further, several aspects of our data replicate known behavioral effects induced by RSC lesions (further described in the General Discussion), again suggesting that the initial two shocks did not markedly influence the outcome of the present experiment.

\section{Discussion}

The RSC is known to contribute to contextual and spatial learning and memory. However, there is a growing body of research indicating that the RSC is also involved in learning and memory for discrete cues. We therefore examined the RSC's contribution to second-order fear conditioning, a form of higher-order conditioning, in which one cue gains the ability to elicit a CR even though it is never directly paired with reinforcement. During Phase 1 of training, one visual stimulus (V1) was repeatedly paired with the reinforcer (i.e., footshock), and the other visual stimulus (V2) was not. Then, in Phase 2, one auditory stimulus (A1) was followed by V1, and a second stimulus (A2) was followed by V2. Since both A1 and A2 were followed by a visual stimulus, any fear conditioned to A1 must have resulted from V1's unique association with the reinforcer, and could not be the result of any primary reinforcing value of the light, or sensitization via repeated presentation of A1. For both Sham and RSC lesioned rats, second-order 
conditioning was observed; as Phase 2 training progressed, there was more conditioned suppression to A1 relative to A2. Thus, pre-training lesions of the RSC had no impact on second-order fear conditioning, suggesting that the RSC is not necessary for some forms of higher-order conditioning.

The finding that lesions of the RSC had no impact on the acquisition/expression of second-order fear conditioning is, to a degree, surprising. Anatomically, the RSC sits at the interface of sensory cortical regions and the parahippocampal-hippocampal memory system. The fact that the RSC receives input from both visual and auditory sensory cortices suggests that it is well-placed to contribute to learning and memory in situations that require processing multiple stimuli from different modalities. Indeed, it has been suggested that the RSC is specifically involved in forming associations between multiple stimuli, or stimulus-stimulus (S-S) learning (e.g., Bucci \& Robinson, 2014). Consistent with this idea, two previous studies from our lab have demonstrated a role for the RSC in a different form of higher-order conditioning, sensory preconditioning (e.g., Robinson et al., 2012, 2014). Thus, the current findings suggest a possible dissociation of RSC involvement in two forms of higher-order conditioning. However, it is important to note that the earlier studies involved appetitive reinforcement, whereas the current experiment involved aversive reinforcement. In addition, caution should be exercised making cross-experiment comparisons. Yet, with those caveats in mind, the findings from several studies examining sensory preconditioning and second-order conditioning suggests a double dissociation between the role of the RSC and the hippocampus. Specifically, manipulations of the RSC impair sensory preconditioning (e.g., Robinson et al., 2012, 2014) and not second-order conditioning (current experiment), whereas 
manipulations of the hippocampus impact second-order conditioning (Gilboa, Sekeres, Moscovitch, \& Winocur, 2014) but not some forms of sensory preconditioning (e.g., Iordanova, Good, \& Honey, 2011; see Honey, Iordanova \& Good, 2014 for a review). Future experiments are required to provide within-experiment comparisons, but the overall pattern suggests differential functions of the RSC and the hippocampus in two forms of higher-order conditioning.

Although the first- and second-order stimuli were presented together in the current study, it is possible that they did not become associated with each other. Secondorder conditioning is often thought to reflect a direct association between the secondorder stimulus and the response evoked by the first order stimulus, traditionally known as S-R learning. This is supported by studies demonstrating that post-training manipulations of the original reinforcer's value, or the value of the first-order conditioned stimulus (e.g., via extinction), often have no effect on responding to the second-order stimulus (e.g., Rescorla, 1973a, b, 1974, 1977, 1982; Rizley \& Rescorla, 1972). Thus, one possibility is that RSC lesions had no impact on second-order conditioning because the subjects associated the stimulus and the response, not the two stimuli. It is worth noting that S-R learning has been observed in a range of second-order conditioning paradigms, including: conditioned suppression (e.g., Rescorla, 1982), contextual fear conditioning (e.g., Helmstetter \& Fanselow, 1989), and appetitive conditioning (e.g., Holland \& Rescorla, 1975; Setlow, Gallagher, \& Holland, 2002).

Nevertheless, in some cases second-order conditioning does result in S-S learning. For example, Rescorla (1982) found that when S1 and S2 were simultaneously presented, extinction of S1 did attenuate conditioned suppression evoked by S2. In this case, S1 and 
S2 appeared to be directly associated (i.e., S-S learning). In contrast, extinction of S1 had no impact on S2 when the two stimuli were trained sequentially (i.e., S-R learning), as in the current experiment. Since the current experiment did not independently verify the associative structure underlying second-order conditioning, future experiments are required to determine if $\mathrm{RSC}$ involvement is dependent upon a particular associative structure. However, it should be noted that two features of the current experiment are consistent with second-order conditioning resulting in S-R learning. First, as described, sequential presentation of the first- and second-order stimuli seems to promote S-R learning. Second, Rescorla (1982) has demonstrated that in the simultaneous secondorder conditioning procedure, where S-S learning occurs, extended exposure to S1 (either reinforced or non-reinforced) during Phase 1 prevented extinction of S1 from affecting responding to $\mathrm{S} 2$. One interpretation of this finding is that stimulus $(\mathrm{S})$ and response $(\mathrm{R})$ features of S1 might compete for association with S2, and that prior exposure to S1 reduces its ability to enter into an S-S association, giving way to an S-R structure (Rescorla, 1982; see also Rescorla, 1977). It is interesting to note that the present experiment included 48 total reinforced presentations of V1. In contrast, Rescorla (1982, Experiments 3 and 4) compared groups that received either 12 or 2 presentations of S1. In that experiment, 12 presentations of S1 produced S-R learning, whereas 2 presentations of S1 produced S-S learning. Thus, the extensive V1 exposure in the present experiment seems especially likely to produce $\mathrm{S}-\mathrm{R}$, rather than $\mathrm{S}-\mathrm{S}$, learning. Overall this analysis predicts that if the RSC contributes to S-S, but not S-R learning, then second-order conditioning parameters that promote S-S learning should be sensitive to RSC manipulation. This prediction awaits further study. 
Although lesions of the RSC did not impact second-order fear conditioning, they did attenuate first-order discrimination learning. Early in first-order conditioning all rats rapidly suppressed responding to both V1+ and V2-. However, Sham lesioned rats extinguished responding to V2- much faster than the RSC lesioned rats. This deficit in first-order discrimination learning is consistent with at least two reports that RSC lesions impair performance on conditional visual discrimination tasks (Bussey, Muir, Everitt, \& Robbins, 1996, 1997; cf. Nelson, Hindley, Haddon, Vann, \& Aggleton, 2014) and suggests that the null effect of RSC lesions on second-order conditioning cannot be attributed simply to ineffective lesions. Indeed, the extent of lesion damage here was similar to that reported in prior studies in which impairments were observed in both appetitive and aversive conditioning paradigms (e.g., Keene \& Bucci, 2008a, b, c; Robinson et al., 2012).

Given the reciprocal connections with the RSC and visual cortex, it is possible that RSC lesions disrupted detailed sensory processing of the visual stimuli. It should also be noted that although both groups of rats discriminated V1 from V2 prior to the start of the second-order conditioning phase, the generalization early in training may have resulted in stronger V1 conditioning for RSC compared to Sham rats. If so, this may have occluded any possible attenuation of second-order conditioning in RSC-lesioned rats. However, this possibility seems unlikely given that extinction of V1 progressed at the same rate for both groups (Figure 7). Over the entire response scale, there were no detectable differences in the rate of extinction of V1, suggesting that V1 had acquired a similar level of conditioning during the first-order phase. Thus, the failure of RSC lesions 
to impact second-order conditioning appears to be independent of the first-order conditioning deficit.

Relatedly, it could be argued that effects of RSC lesions observed here during first-order conditioning but not second-order conditioning simply reflect involvement of RSC in learning visual discriminations but not auditory discriminations, rather than sparing of second order conditioning per se. However, this also seems unlikely for several reasons. First, in addition to its connections with visual cortical areas, RSC is also interconnected with regions of auditory cortex (Todd et al, in press; Vogt \& Miller, 1983), thus it is entirely possible that RSC lesions could impact conditioning involving auditory cues. Consistent with this, two recent studies have demonstrated that manipulations of RSC can affect first order conditioning involving auditory cues (trace fear conditioning with an auditory CS; Kwapis et al. 2015; remote retrieval of delay conditioned auditory cues, Todd et al., in press). More importantly, there is also evidence that RSC damage can impair first-order discrimination learning with auditory stimuli (Gabriel et al, 1987). Thus, RSC lesions can indeed affect discriminations involving two auditory cues under certain conditions. Together, these findings suggest that the pattern of results observed in the current study cannot be attributed merely to an effect of RSC on visual versus auditory discrimination learning.

In sum, pre-training lesions of the RSC had no impact on second-order fear conditioning, but did attenuate first-order discrimination learning. Although the RSC is anatomically well positioned to co-jointly process multiple stimuli, it appears it is not necessary for this form of higher-order conditioning. Based in part on studies of sensory preconditioning, a different form of higher-order conditioning, it has been suggested that 
the RSC is crucial for S-S associations (e.g., Bucci \& Robinson, 2014). We suggest the possibility that the RSC is not necessary for second-order conditioning, because this form of higher-order conditioning often results in S-R learning. 


\section{References}

Brogden, W. J., (1939). Sensory preconditioning. Journal of Experimental Psychology, $25,323-332$.

Bucci, D. J., \& Robinson, S. (2014). Toward a conceptualization of retrohippocampal contributions to learning and memory. Neurobiology of Learning and Memory, 116, 197-207.

Bussey, T. J., Muir, J. L., Everitt, B. J., \& Robbins, T. W. (1996). Dissociable effects of anterior and posterior cingulated cortex lesions on the acquisition of a conditional visual discrimination: Facilitation of early learning vs. impairment of late learning. Behaviorual Brain Research, 82, 45-56.

Bussey, T. J., Muir, J. L., Everitt, B. J., \& Robbins, T. W. (1997). Triple dissociation of anterior cingulated, posterior cingulated, and medial frontal cortices on visual discrimination tasks using a touchscreen testing procedure for the rat. Behavioral Neuroscience, 111, 920-936.

Corcoran, K. A., Donnan, M. D., Tronson, N. C., Guzmán, Y. F., Gao, C., Jovasevic, V., \& Radulovic, J. (2011). NMDA receptors in retrosplenial cortex are necessary for retrieval of recent and remote context fear memory. The Journal of Neuroscience, $31,11655-11659$.

Czajkowski, R., Jayaprakash, B., Wiltgen, B., Rogerson, T., Guzman-Karlsson, M. C., Barth, A. L., Trachtenber, J. T., \& Silva, A. J. (2014). Encoding and storage of spatial information in the retrosplenial cortex. Proceedings of the National Academy of Sciences, 111(23), 8661-8666. 
Gabriel, M., Lambert, R. W., Foster, K., Orona, E., Sparenborg, S., \& Maiorca, R. R. (1983). Anterior thalamic lesions and neuronal activity in the cigulate and retrosplenial cortices during discriminative avoidance behavior in rabbits. Behavioral Neuroscience, 97, 675-696.

Gabriel, M., Sparenborg, S.P., \& Stolar, N. (1987). Hippocampal control of cingulate cortical and anterior thalamic information processing during learning in rabbits. Experimental Brain Research, 67, 131-152.

Gilboa, A., Sekeres, M., Moscovitch, M., \& Winocur, G. (2014). Higher-order conditioning is impaired by hippocampal lesions. Current Biology, 24, 22022207.

Helmstetter, F. J., \& Fanselow, M. S. (1989). Differential second-order aversive conditioning using contextual stimuli. Animal Learning \& Behavior, 17, 205-212.

Holland, P. C., \& Rescorla, R. A. (1975). Second-order conditioning with food unconditioned stimulus. Journal of Comparative and Physiological Psychology, $88,459-467$.

Honey, R. C., Iordanova, M. D., \& Good, M. (2014). Associative structures in animal learning: dissociating elemental and configural processes. Neurobiology of Learning and Memory, 108, 96-103.

Iordanova, M. D., Good, M., \& Honey, R. C. (2011). Retrieval-mediated learning involving episodes requires synaptic plasticity in the hippocampus. The Journal of Neuroscience, 11, 7156-7162. 
Keene, C. S., \& Bucci, D. J. (2008a). Contributions of the retrosplenial and posterior parietal cortices to cue-specific and contextual fear conditioning. Behavioral Neuroscience, 122, 89-97.

Keene, C. S., \& Bucci, D. J. (2008b). Involvement of retrosplenial cortex in processing multiple conditioned stimuli. Behavioral Neuroscience, 122, 651-658.

Keene, C. S., \& Bucci, D. J. (2008c). Neurotoxic lesions of retrosplenial cortex disrupt signaled and unsignaled contextual fear conditioning. Behavioral Neuroscience, 122, $1070-1077$.

Kwapis, J. L., Jarome, T. J., Lee, J. L., Gilmartin, M. R., \& Helmstetter, F. J. (2014). Extinguishing trace fear engages the retrosplenial cortex rather than the amygdala. Neurobiology of learning and memory, 113, 41-54.

Kwapis, J. L., Jarome. T. J., Lee, J. L., \& Helmstetter, F. J. (2015). The retrosplenial cortex is involved in the formation of memory for context and trace fear conditioning. Neurobiology of Learning and Memory, 123, 110-116.

Miller, A. M. P., Vedder, L. C., Law, M., \& Smith, D. M. (2014). Cues, context, and long-term memory: the role of the retrosplenial cortex in spatial cognition. Frontiers in Human Neuroscience, 8, 1-15.

Nelson, J. B., Sanjuan, M. C., Vadillo-Ruiz, S., Pérez, J., \& León, S. P. (2011). Experimental renewal in human participants. Journal of Experimental Psychology: Animal Behavior Processes, 37, 58-70.

Nelson, A. J. D., Hindley, E. L., Haddon, J. E., Vann, S. D., \& Aggleton, J. P. (2014). A novel role for the rat retrosplenial cortex in cognitive control. Learning \& Memory, 21, 90-97. 
Rescorla, R. A. (1973a). Second-order conditioning: Implications for theories of learning. In Contemporary approaches to conditioning and learning (eds. F. J. McGuigan \& E. B. Lumsden). Washington, D. C. Winston.

Rescorla, R. A. (1973b). Effect of US habituation following conditioning. Journal of Comparative and Physiological Psychology, 82, 137-143.

Rescorla, R. A. (1974). Effect of inflation of the unconditioned stimulus value following conditioning. Journal of Comparative and Physiological Psychology, 86, 101106.

Rescorla, R. A. (1977). Pavlovian second-order conditioning: Some implications for instrumental behavior. In Operant-Pavlovian interactions (eds. H. Davis \& H. M. B. Hurwitz), pp. 133-164. Hillsdale, New Jersey: Erlbaum.

Rescorla, R. A. (1980). Pavlovian second-order conditioning: Studies in associative learning. Hillside, N. J.: Erlbaum.

Rescorla, R. A. (1982). Simultaneous second-order conditioning produces S-S learning in conditioned suppression. Journal of Experimental Psychology: Animal Behavior Processes, 8, 23-32.

Rescorla, R. A., \& Cunningham, C. L. (1978). Within-compound flavor associations. Journal of Experimental Psychology: Animal Behavior Processes, 4, 267-275.

Rizley, R. C., \& Rescorla, R. A. (1972). Associations in second-order conditioning and sensory preconditioning. Journal of Comparative and Physiological Psychology, $81,1-11$. 
Robinson, S., Keene, C. S., Iaccarino, H. F., Duan, D., \& Bucci, D. J. (2012).

Involvement of retrosplenial cortex in forming associations between multiple sensory stimuli. Behavioral Neuroscience, 125, 578-587.

Robinson, S., Todd, T. P., Pasternak, A. R., Luikart, B. W., Skelton, P. D., Urban, D. J., \& Bucci, D. J. (2014). Chemogenetic silencing of neurons in the retrosplenial cortex disrupts sensory preconditioning. The Journal of Neuroscience, 34, 1098210988.

Setlow, B., Gallagher, M., \& Holland, P. C. (2002). The basolateral complex of the amygdala is necessary for acquisition but not expression of CS motivational value in appetitive Pavlovian second-order conditioning. European Journal of Neuroscience, 15, 1841-1853.

Sugar, J., Witter, M. P., van Strien, N. M., \& Cappaert, N. L. (2011). The retrosplenial cortex: intrinsic connectivity and connections with the (para)hippocampal region in the rat. An interactive connectome. Frontier in Neuroinformatics, 5, 7.

Todd, T. P., \& Bucci, D. J. (2015). Retrosplenial cortex and long-term memory: Molecules to behavior. Neural Plasticity, 414173, 1-9.

Todd, T.P., Mehlman, M.L., Keene, C.S., DeAngeli, N.E., and Bucci, D.J. (in press) Retrosplenial cortex is required for the retrieval of remote memory for auditory cues. Learning and Memory.

van Groen, T., \& Wyss, J. M. (1990). Connections of the retrosplenial granular a cortex in the rat. Journal of Comparative Neurology, 300, 593-606.

van Groen, T., \& Wyss, J. M. (1992). Connections of the retrosplenial dysgranular cortex in the rat. Journal of Comparative Neurology, 315, 200-216. 
van Groen, T., \& Wyss, J. M. (2003). Connections of the restrosplenial granular b cortex in the rat. Journal of Comparative Neurology, 463, 249-263.

Vann, S. D., Aggleton, J. P., \& Maguire, E. A. (2009). What does the retrosplenial cortex do? Nature Reviews Neuroscience, 10, 792-803.

Vogt, B. A. and Miller, M. W. (1983) Cortical connections between rat cingulate cortex and visual, motor, and postsubicular cortices. The Journal of Comparative Neurology, 216, 192-210.

Wyss, J. M., \& van Groen, T. (1992). Connections between the retrosplenial cortex and the hippocampal formation in the rat: A review. Hippocampus, 2, 1-12. 


\section{Acknowledgments}

This work was supported by National Science Foundation Grants IOS1353137 and IOS0922075 (D.J.B.) and National Institutes of Health Grant MH105125 (T.P.T.). We thank Dr. Robert N. Leaton and for commenting on an earlier version of this manuscript. In addition, we thank Matthew Y. Jiang and Max L. Mehlman for technical assistance. 


\section{Table 1}

Experimental Design.

\begin{tabular}{llll}
\hline Pre-exposure & $\begin{array}{l}\text { First-order } \\
\text { conditioning }\end{array}$ & $\begin{array}{l}\text { Second-order } \\
\text { conditioning }\end{array}$ & Extinction \\
\hline V1-, V2-, A1-, A2- & V1+, V2- & A1 $\rightarrow$ V1, A2 $\rightarrow$ V2 & V1-
\end{tabular}

Note. V1 and V2 were flashing light and panel light, counterbalanced. A1 and A2 were noise and clicker, counterbalanced. "“+" = $1 \mathrm{~mA}, 1 \mathrm{~s}$ footshock. "_" = nonreinforcement. All stimuli were $30 \mathrm{~s}$ in duration. During the second-order conditioning phase, stimuli were presented sequentially. See text for additional details. 


\section{Table 2}

Stereotaxic coordinates for retrosplenial cortex (RSC) lesions.

\begin{tabular}{lll}
\hline AP & ML & DV \\
\hline-2.0 & \pm 0.3 & -2.0 and -2.7 \\
-3.5 & \pm 0.4 & -2.0 and -2.7 \\
-5.0 & \pm 0.4 and \pm 1.0 & -2.0 and -2.7 (medial site) and -2.0 (lateral site) \\
-6.5 & \pm 0.8 and \pm 1.5 & -2.0 and -2.8 (medial site) and -3.4 (lateral site) \\
-8.0 & \pm 1.6 and \pm 2.4 & -2.5 (medial site) and -3.1 (lateral site) \\
-9.0 & \pm 3.4 & -4.0 \\
\hline
\end{tabular}

Note. All anterior/posterior (A/P), medial/lateral (M/L) and dorsal/ventral (D/V)

measurements are derived from bregma, midline, and skull surface, respectively (measurements are in $\mathrm{mm}$ ). 
Figure 1

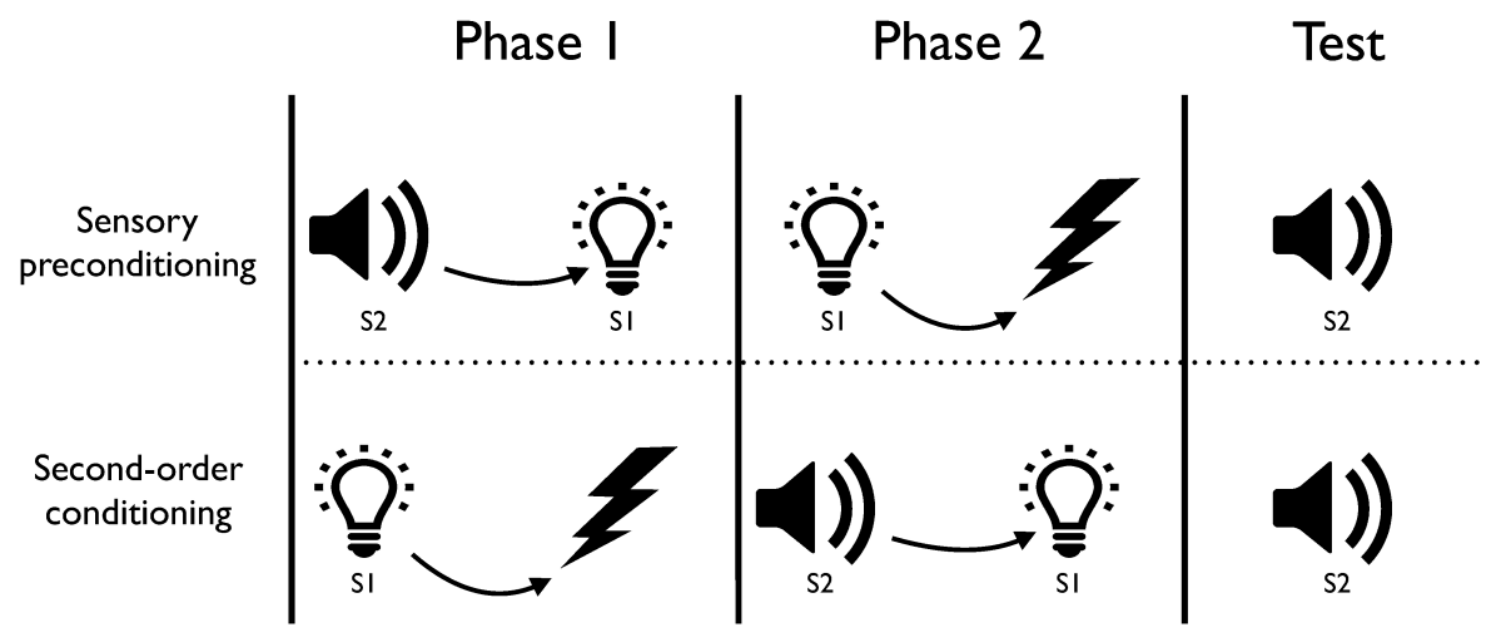

Figure 1. Schematic diagram of sensory preconditioning and second-order conditioning.

In both cases, during testing there is a conditioned response to S2 (i.e., the tone) even though it has never been directly paired with reinforcement (in this case, an aversive footshock). 


\section{Figure 2}

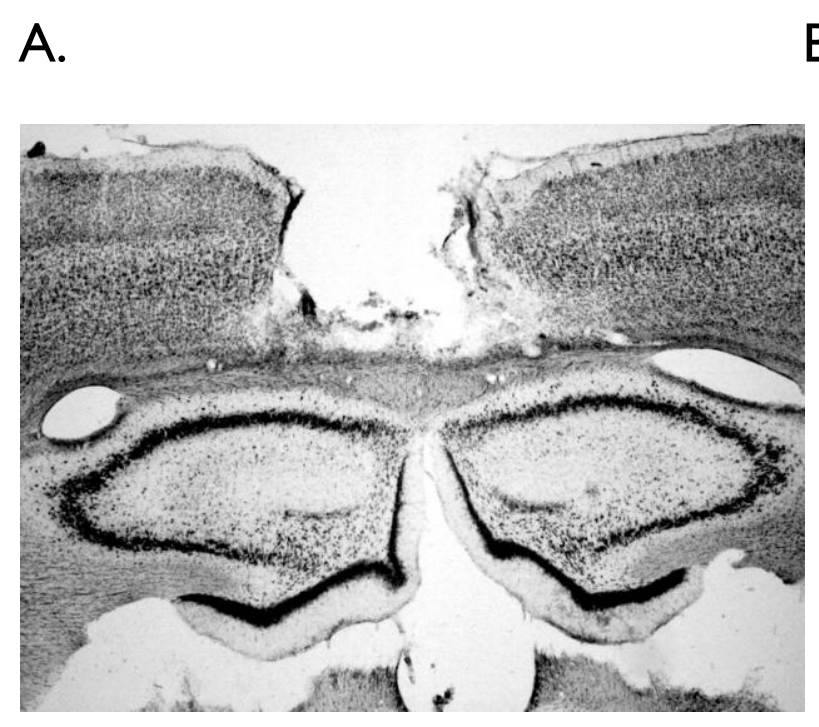

B.
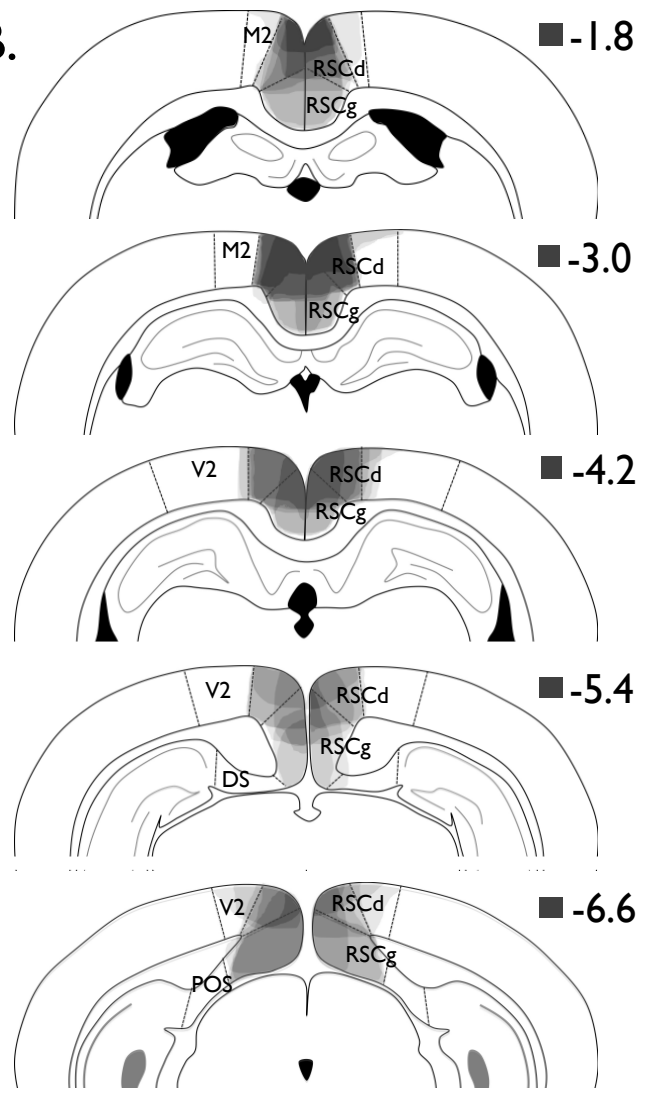

Figure 2. A: Photomicrograph of a representative RSC lesion. B: Drawings of lesions at five levels along the rostro-caudal extent of the $\operatorname{RSC}(-1.8,-3.0,-4.2,-5.4$, and 6.6 posterior to bregma). At each level, lesion drawings were stacked onto a single image. The darkness of an area indicates the number of lesion cases that include that area. Grey boxes (next to the bregma values) represent the expected darkness for overlap from all subjects. M2 = secondary motor cortex, $\mathrm{RSCd}=$ retrosplenial dysgranular, $\mathrm{RSCg}=$ retrosplenial granular, $\mathrm{V} 2=$ secondary visual cortex, DS = dorsal subiculum, POS = postsubiculum. 


\section{Figure 3}

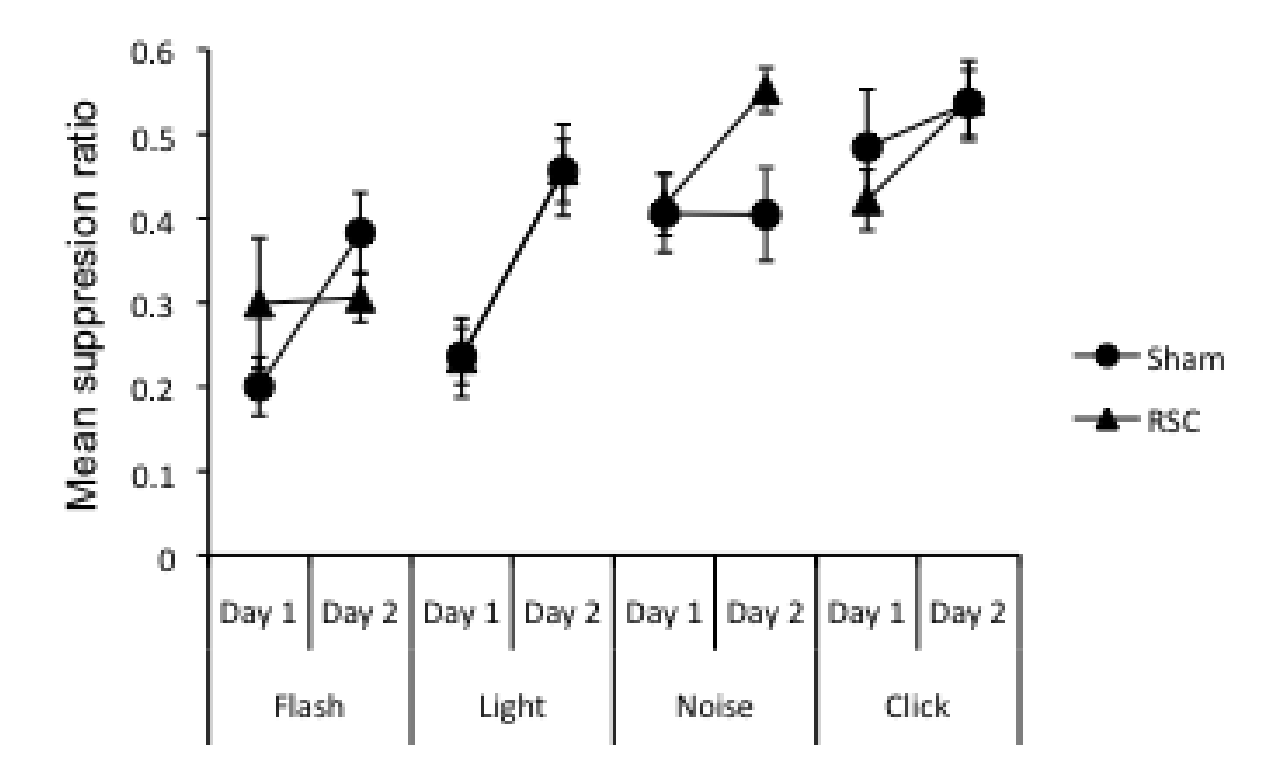

Figure 3. Mean suppression to non-reinforced presentation of the Flash, Light, Noise, and Click during the pre-exposure phase. Each day of pre-exposure consisted of two trials of each stimulus. Sham $=$ sham lesioned rats, $\mathrm{RSC}=$ retrosplenial lesioned rats. Error bars represent $\pm 1 S E M$. 


\section{Figure 4}

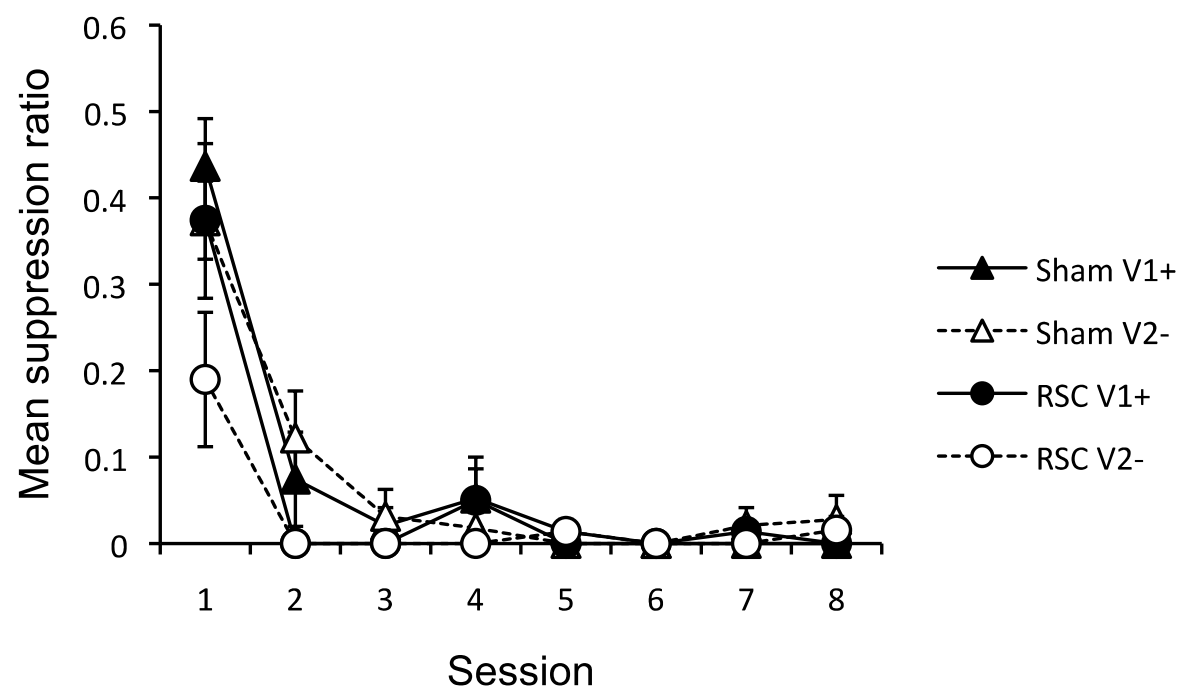

Figure 4. Mean suppression to the first presentation of each visual cue during the first 8 sessions of first-order conditioning. Sham $=$ sham lesioned rats, RSC $=$ retrosplenial lesioned rats. Error bars represent \pm 1 SEM. 


\section{Figure 5}

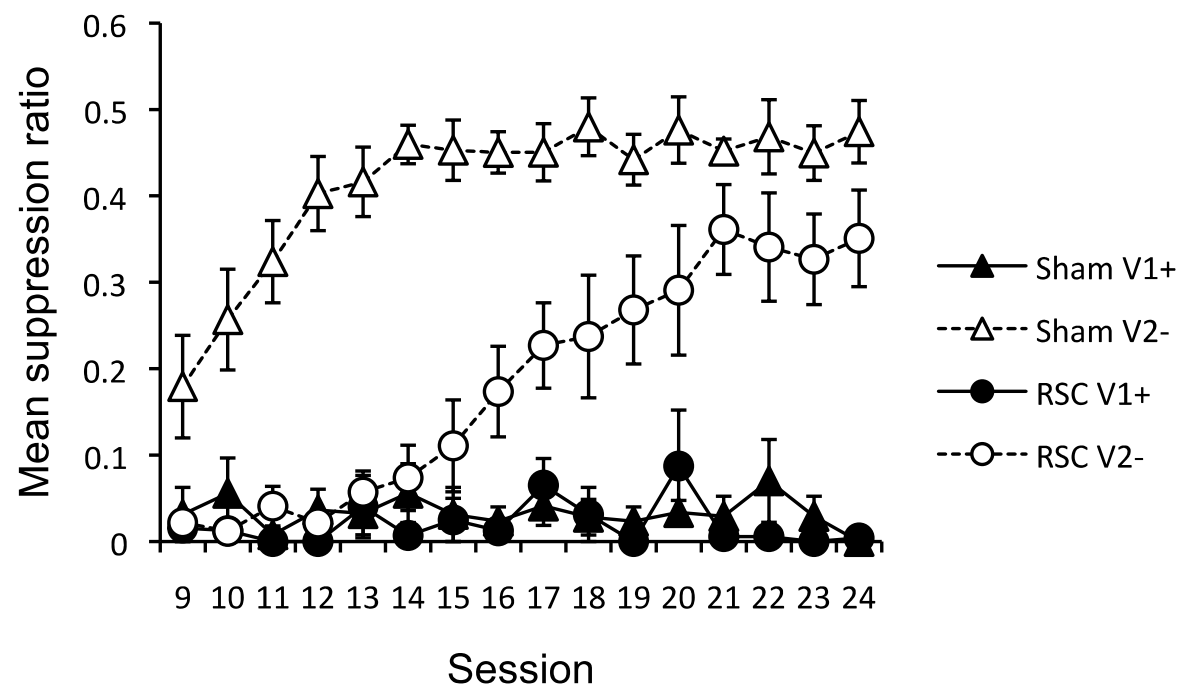

Figure 5. Mean suppression to the visual cues for the final 16 sessions of first-order conditioning. Sham $=$ sham lesioned rats, $\mathrm{RSC}=$ retrosplenial lesioned rats. Error bars represent \pm 1 SEM. 


\section{Figure 6}

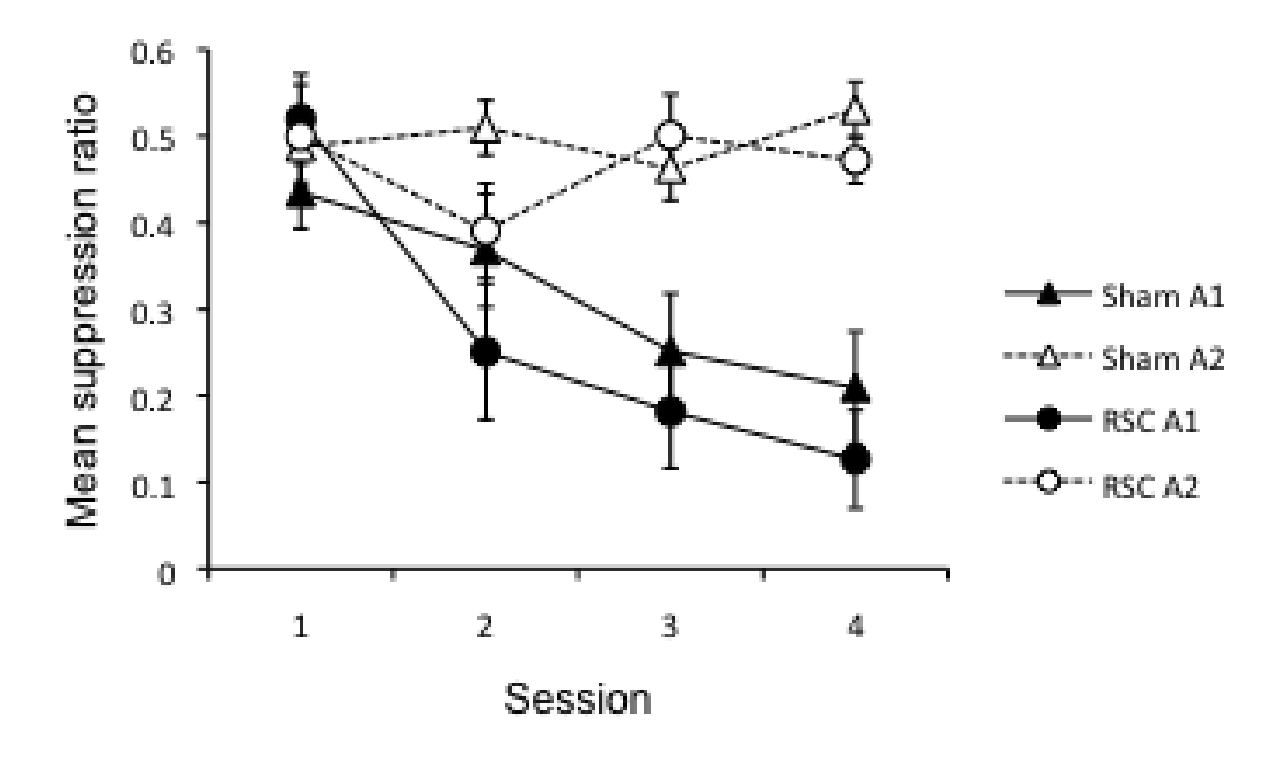

Figure 6. Mean suppression to the auditory cues during the second-order conditioning phase. A1 was followed by V1, and A2 was followed by V2. Sham = sham lesioned rats, $\mathrm{RSC}=$ retrosplenial lesioned rats. Error bars represent $\pm 1 S E M$. 


\section{Figure 7}

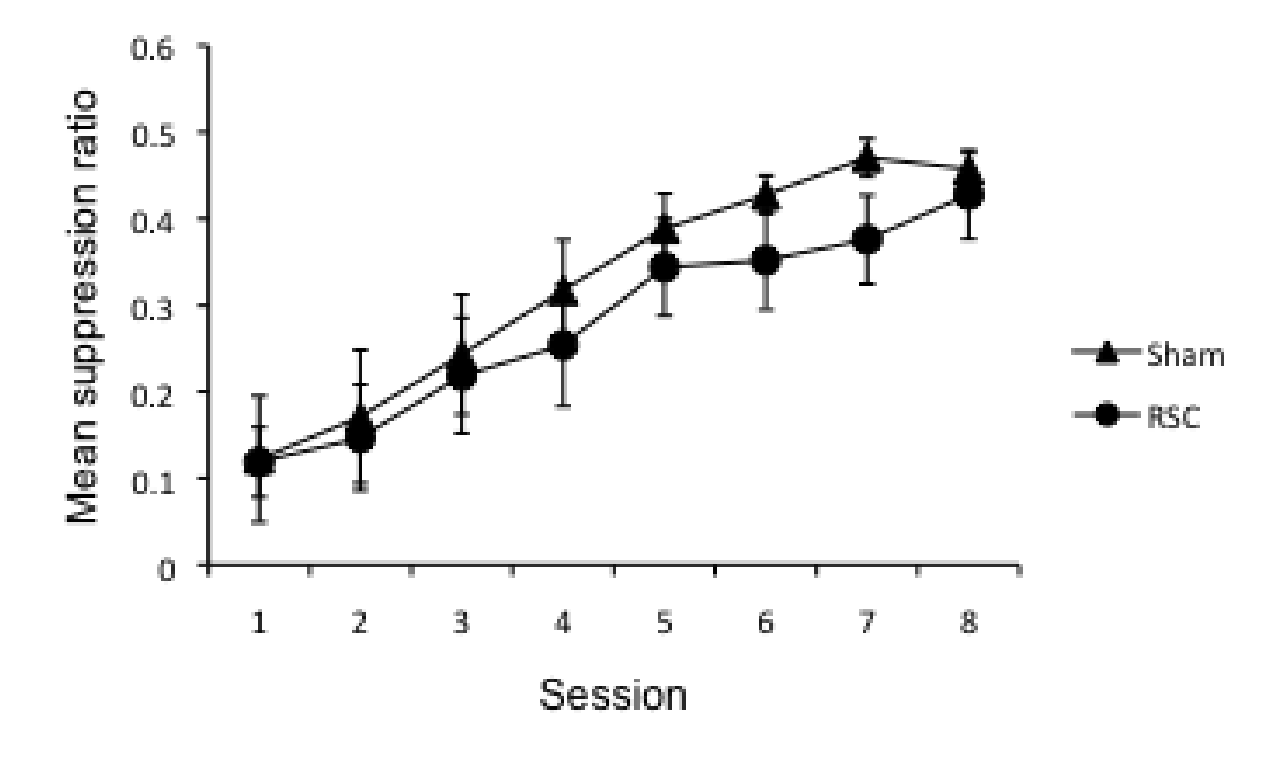

Figure 7. Mean suppression during extinction of the first-order V1+. During extinction, there were 16 non-reinforced presentations of V1 for 8 consecutive sessions. Sham $=$ sham lesioned rats, $\mathrm{RSC}=$ retrosplenial lesioned rats. Error bars represent \pm 1 SEM. 